\title{
Canadian
} Science Publishing

Canadian Journal of Physics

Revue canadienne de physique

\section{STEREOTACTIC RADIOSURGERY DELIVERY VERIFICATION USING TETRAZOLIUM SALT-BASED GEL AS A DOSIMETER}

\begin{tabular}{|r|l|}
\hline Journal: & Canadian Journal of Physics \\
\hline Manuscript ID & cjp-2017-0067.R1 \\
\hline Manuscript Type: & Article \\
\hline Date Submitted by the Author: & 02-Mar-2017 \\
\hline Complete List of Authors: & $\begin{array}{l}\text { Courter, Erik; Wright State University, Department of Physics } \\
\text { Gossman, Michael; Regulation Directive Medical Physics, ; Tri-State } \\
\text { Regional Cancer Center, }\end{array}$ \\
\hline $\begin{array}{r}\text { Klease Select from this Special } \\
\text { Issues list if applicable: }\end{array}$ & \\
\hline \hline & \\
\hline
\end{tabular}

\section{SCHOLARONE}

Manuscripts 


\title{
5 STEREOTACTIC RADIOSURGERY DELIVERY VERIFICATION USING TETRAZOLIUM
}

\section{SALT-BASED GEL AS A DOSIMETER}

\author{
${ }^{1,2,3}$ MICHAEL S. GOSSMAN and ${ }^{3}$ ERIK J. L. COURTER
}

10

${ }^{1}$ Regulation Directive Medical Physics, 104 Hildeen Court, Russell, KY 41101, USA;

${ }^{2}$ Tri-State Regional Cancer Center, Radiation Oncology Department, $70623^{\text {rd }}$ Street, Ashland, KY 41101, USA;

${ }^{3}$ Wright State University, Department of Physics, 248 Fawcett Hall, 3640 Colonel Glenn Highway, Dayton, OH 45435, USA msgossman@hotmail.com erikjlcourter@yahoo.com

ABSTRACT - Objective: The recently made available ClearView $^{\circledR}$ tetrazolium salt-based gel dosimeter, encapsulated in a vial, was tested to determine feasibility in use as an end-to-end dosimeter for stereotactic radiosurgery commissioning and quality assurance. Methods: A fabricated adaptor enabled direct attachment of the gel vial lid to a rod extension. The rod was inserted into a water-filled head phantom at the open neck location and locked into position. Once quality assurance testing of the linear accelerator was completed, a stereotactic plan was deployed. Results: Optical scanning software enabled a direct comparison of the measured positional isocenter and dose distribution to the intended plan. Delivery accuracy was verified as line-dose profile plots of measured data coincide with the planned dose location and distribution. Conclusions: It has been demonstrated here that ClearView $^{\circledR}$ gel contained in a vial is a suitable dosimeter for verifying stereotactic radiation therapy delivery accuracy. The end-to-end testing provided is geared to work identically for any small field study, 
including stereotactic radiosurgery and radiotherapy, volumetric modulated arc therapy, intensity modulated radiation therapy, and three-dimensional conformal radiation therapy.

Short Title: $\quad$ SRS end-to-end verification with an in-phantom gel dosimeter

Keywords: $\quad$ Fricke, gel, optical density, radiosurgery, stereotactic

35 PACS Numbers: $\quad$ 42.25.Bs, 78.40.Dw, 07.57.Kp, 29.40.-n, 85.25.Pb, 87.55.-x, 87.55.-x

Corresponding Author: Michael S. Gossman, M.S., DABR, FAAPM, Regulation Directive Medical Physics, 104 Hildeen Court, Russell, KY 41101, USA, (606) 329-0060, msgossman@hotmail.com

40 Conflicts of Interest:

We have no conflicts of interest to report for this research. No funding has been provided at any capacity for this study.

\section{INTRODUCTION}

45 As a part of the commissioning process, it is recommended that the quality assurance program include an end-to-end test that mimics a patient treatment. ${ }^{1}$ The end-to-end test is intended to assure that all the components work together to yield the desired dose distribution. Such testing considers all aspects of treatment preparation, simulation, planning, quality assurance, imaging verification, delivery, and analysis. The uncertainties throughout are vital to be recognized and critical to the validation of

50 treatment delivery systems. Here, we consider use of the newly available Modus Medical Devices, Inc. (Ontario, CANADA) Model ClearView ${ }^{\circledR}$ gel dosimeter as an advanced radiation detector. 
ClearView $^{\circledR}$ gel is similar to current radiochromic gels in that, upon irradiation, physical color changers in the gel are present. These color changes are proportional to the dose received by the gel, thus retaining

55 the dose information after dose delivery is complete. The resulting color concentration can then be measured as an optical density (OD) using optical computed tomography (CT). However, ClearView ${ }^{\circledR}$ offers some notable advantages over standard Fricke radiochromic gels. Unlike Fricke gels, ClearView ${ }^{\circledR \prime} \mathrm{s}$ tetrazoleum salt formula does not suffer from spatial diffusion common in xylenol orange gels which causes a loss of spatial dose information over time..$^{2-3}$ ClearView $^{\circledast}$ also has the capability to measure 60 doses of up to $80 \mathrm{~Gy}$, where only doses of up to $40 \mathrm{~Gy}$ are measureable with specific oxygen-purged Fricke gels. ${ }^{4}$ The main advantage that both $\mathrm{ClearView}^{\circledR}$ and polymer gels share over Fricke radiochromic gels is the absence of spatial diffusion which causes errors in dose distribution. ${ }^{5-6}$ Additionally, most polymer gels display properties such as high reproducibility, well-defined spatial distribution, and low dose-rate dependence, which are favorable for SRS QA. ${ }^{7}$ However, polymer gel dosimeters are not

65 without problems of their own. Additionally, polymeric radicals used to report dose in polymer gel dosimeters cannot diffuse and terminate, causing additional reported dose after irradiation has been completed. ${ }^{8}$ While polymer gels all rely on the cross-linking of acrylic monomers in place of Fricke ionization, differences in monomer composition can largely affect the performance of polymer gels. Polyacrylamide BANG and PAG gels are easily contaminated by oxygen, requiring the gels to be manufactured, irradiated, and measured in oxygen-free environments. Although normoxic polymer gels do not suffer from this oxygenation problem, they have been shown to have inferior radiation properties when compared to the original PAG gels. ${ }^{9-10}$ Different monomer compositions can also pose problems for use such as toxicity as well as carcinogenic properties. ${ }^{8,10}$

75 It is conclusive then that advantages in the use of ClearView ${ }^{\circledR}$ gel include three dimensional resolution, high spatial resolution, tissue equivalence, reproducibility, chemical stability. The known remaining 
disadvantages are that the scanner is quite expensive, as it is a modern product with advanced software associated with it. It is projected that most centers will find the system too expensive to have on-site, which therefore requires the shipping of samples with no real-time result. With shipping necessary, it is

80 important that the user adhere to the refrigeration guidelines of the manufacturer, to prevent the formation of biological contaminants.

Properties of chemical dosimetry can be traced back as far as 1927 with works by Fricke. ${ }^{11-12}$ At that time, chemical reactions of ferrous sulphate solutions were being examined, due to their inherent

85 properties of change when irradiated. A Fricke gel dosimeter is characteristically $89 \%$ water and $5 \%$ gelatin-agarose, with the remainder usually as the ferrous chemical that changes when irradiated. An example would be a concentration of xylenol orange, where $\mathrm{Fe}^{2+}$ ions change to $\mathrm{Fe}^{3+}$ ions upon irradiation..$^{3,13}$ In a notable dissertation by Fried in 1995, a more advanced Fricke-type gel, yet still an adolescent form of the xylenol orange gel, was presented. ${ }^{14}$ Upon irradiation, the color change in the gel

90 (radiochromic) signal led to a change in the optical density (OD) proportional to the absorbed dose. He provided a premise for the change in the form of Beer's Law, stating an increase in the solution's concentration change between ferric ions and xylenol orange was proportional to an increase in the attenuated optical path length of light through the solution. ${ }^{14-15}$ From this relationship, we can relate the OD to the absorbed dose given. However, Fricke-based gels suffer from multiple issues related to the 95 chemical stability of the gel and also the chemical and spatial stability of the dose-image. ${ }^{3,13}$

In this evaluation, we examine the newly developed ClearView ${ }^{\circledR}$ gel dosimeter, which is based on the previously established radiochromic properties of tetrazolium salts. Tetrazolium salts were first developed for liquid and film dosimetry. One characteristic of the new radiochromic system is a strong linearity between OD and absorbed dose through cumulative doses of 5,000 cGy and with rapid dose- 
rate deliveries of 6,000 cGy/min. ${ }^{16,17}$ Some suggest that tetrazolium salt-based dosimeters may overcome radiation detector limitations in spatial resolution and profile accuracy known to be exhibited by diamond detectors, ion chambers, and diodes. ${ }^{17}$ This progress has been made possible with modern advancements to the classic system for optical analysis. The current means of performing optical density

105 measurements is through the use of a cone beam optical computed tomography (CT) scan. This method allows assessment of scattering and absorption through the gel vials by experiment. From this preliminary data, the gel detector can then be reengineered to produce the most useful chemical form, thus providing the most ideal detector. ${ }^{18-20}$ Gel detectors offer a unique ability to provide dose profiles based on the physical process of light transmission through the molecules of the gel. Therefore, gel

110 dosimetry profile generation can be conducted using vastly more data points than for a solid detector of any type. Our aim in this trial was to test a tetrazolium salt-based gel dosimeter, with proprietary chemistry, as a vial containable radiation detector. We sought to apply emphasis on the need to examine the geometric and dosimetric accuracy in delivery for perhaps the most difficult radiation oncology treatment modality available: stereotactic radiosurgery.

\section{MATERIALS \& METHODS}

Dosimetry for this study was conducted using ClearView ${ }^{\circledR}$ gel, which is formed on the basis of gellan gum (1.25 wt. \%) as the gelling agent, suspended in glycerol (10 vol. \%) in water as well as a proprietary

120 tetrazolium salt $(<0.1 \%)$. The net physical density of the gel is similar to that of water at $1.02 \mathrm{~g} / \mathrm{cm}^{3}$. The result of irradiation of the gel is the creation of a purple-colored formazan dye that remains suspended in the gel, observable to the human eye. A darker the shade of the purple color corresponds to a greater amount of chemical reduction in the gel, which in turn corresponds to a higher radiation dose. Since the color of the gel is permanently altered within the gel, geometric and dosimetric characteristics of the 
125 delivered ionizing radiation are retained after irradiation. The manufacturer of the gel claims that the signal is spatially and chemically stable; however, the gel may be subject to biological pollutants. In light of this possibility, it is important for the user to conduct analysis in a time-efficient manner.

The Modus Medical Devices, Ltd. Model Vista ${ }^{\circledR}$ View 3D Visualization Software version 0.3.3 (2015)

130 makes use of cone beam computed tomography technology (Vista ${ }^{\circledR} 15$ scanner) for a three-dimensional rendering of the gel using a 620-633 nm (orange-dark orange) photon light source with a narrow bandwidth. ${ }^{18}$ The change in optical density (OD) of the gel after irradiation is then directly proportional to the absorbed dose, using only the proportionality constant and the linear attenuation coefficient. Together, these properties relate the chemical reduction sensitivity of the salt colloid to the amount of 135 energy absorbed by the gel. From this relationship, only the determination of OD from the visible light CT scanner remains. ${ }^{14,18}$ ClearView $^{\circledR}$ gels were scanned before shipping by the manufacturer to establish a baseline and independent calibration for each vial.

Once the gel vial was received and unpackaged from the manufacturer's ice-packed container, the gel

140 vial was permitted to warm to room temperature for 8 hours before use. Each vial was $9.0 \mathrm{~cm}$ long, 4.3 $\mathrm{cm}$ in diameter, and has a wall thickness of $1.0 \mathrm{~mm}$. Localization fiducials were placed non-coplanar at three different locations on the surface of the vial. The metal fiducial spheres were expected to easily make their location known at the time of gel vial scanning, where triangulation of the scanning geometry to the planned geometry would be possible. In order to enable mounting of the vial, a small

145 adaptor was fabricated out of acrylic. The adaptor was simple in form, being flat at one end and threaded on the opposite end. To secure the gel vial into a stationary position, the acrylic adaptor was mounted to the lid of the gel vial with a strong adhesive. After the glue cured, the opposite end of the 
adaptor was threaded onto a rod used specifically with a head phantom having one of the more advanced stereotactic designs. ${ }^{21}$ Figure 1 illustrates the resulting fabrication and connections.

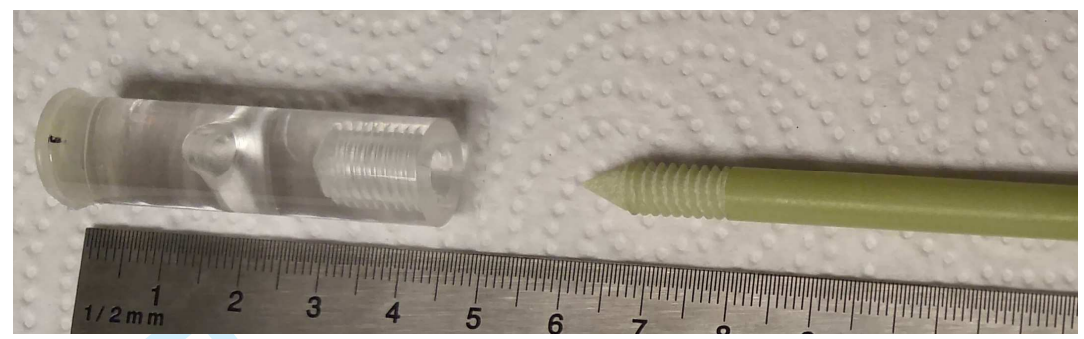

Figure 1. Fabrication result for the gelatin vial and phantom connection rod

The Phantom Laboratory, Inc. ${ }^{\circledR}$ (Salem, NY) Model TLP290 RSVP Phantom ${ }^{\circledR}$ II head phantom (with bite

155 block) features a human-like plastic head that can be filled with water. ${ }^{22}$ It accommodates any mask and bite block-based stereotactic radiotherapy (SRT) application including masked-base treatment and halobased direct mounting of a radiosurgery head frame using cranial screws. The neck of the phantom permits insertion of an ionization detector if desired. Alternatively, as used here, the neck locking system can also be used to hold a head phantom rod in place, where a vial of gel acts as the radiation

160 detector. Once the rod was locked tightly, the position of the gel vial was fixed without further movement. Placement of the vial within the head phantom was adjusted to approximate the existence of a left-sided acoustic neuroma. ${ }^{23}$

An SRS halo was then secured using cranial screws and mounted to the couch of a General Electric ${ }^{\circledR}$

165 (Fairfield, CT) Model LightSpeed ${ }^{\circledR}$ RT Computerized Tomography (CT) scanner. Localization fiducials were then placed on the surface of the head phantom in order to identify the user origin for treatment planning. Resembling the geometry of a patient, the three fiducials were positioned co-planar at locations lateral and anterior along the transverse axis using calibrated wall lasers. A helical CT scan was 
conducted using a stereotactic technique that included $120 \mathrm{kVp}$ x-ray energy at $30 \mathrm{mAs}$ with a $1.25 \mathrm{~mm}$

170 couch increment per slice. Images were then networked to a computer for treatment planning.

We made use of the Varian Medical Systems, Inc. ${ }^{\circledR}$ (Palo Alto, CA) Model Eclipse ${ }^{\circledR}$ treatment planning system version 11.0. The image data set geometry was set at origin using the co-planar fiducials observed on the surface of the head phantom. The vial containing the gel was identified and delineated

175 by contour tools from the base of the vial to the neck where the lid connected. This defined region is uniform in shape is the only region valid for optical scanning of the gel. In the Cone Planning workspace of Eclipse, a single isocenter was placed at the approximate centroid of the vial volume. A $15 \mathrm{~mm}$ cone and 4 arbitrarily chosen SRS Arc beams were applied from a Varian Model TrueBeam ${ }^{\circledR}$ linear accelerator. Each beam had an x-ray energy of 6 MV-FFF assigned to that isocenter and can be described by IEC 6-

1801217 standard geometry with static table positions (T) and gantry (G) rotations. ${ }^{24}$ Specifically, these

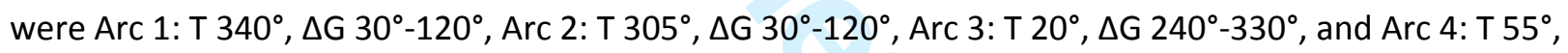
$\Delta \mathrm{G} 240^{\circ}-330^{\circ}$. For a planned dose of $40 \mathrm{~Gy}$ using equally weighted beams, the resulting beam-on time in monitor units for each were $1819 \mathrm{MU}, 1818 \mathrm{MU}, 1462 \mathrm{MU}$, and $1574 \mathrm{MU}$ respectively. A handcalculation data check was performed to insure the accuracy of the results prior to delivery. Treatment

185 planning was completed after programming the Varian Aria ${ }^{\circledR}$ record-and-verify system to include an accompanying cone-beam CT (CBCT) to be performed prior to the SRS delivery. Image registration was expected to correct for any inaccuracies in the raw alignment of the phantom that differed from planned geometry once mounted to the couch of the accelerator. This adjustment was made simple using the six-degree of freedom (6DOF) couch.

The integrity of the linear accelerator isocenter was determined by various imaging modalities. Isocenter checks included the placement of a $(5 \mathrm{~cm})^{3}$ cube, having in internally centered fiducial, on the couch of 
the accelerator. The cross-hairs of the cube were aligned to the light field of the accelerator, then anteriorly imaged using a $6 \mathrm{MV}$ beam by the electronic portal imaging device (EPID) as well as using a

195 laterally projected $120 \mathrm{kVp}$ beam by the On-board Imager. ${ }^{25-27}$ Following that check, a CBCT scan was performed with the OBI. Finally, a Winston-Lutz procedure was introduced for further quality assurance (QA).$^{28}$ This process makes use of the cube and EPID again. However, images are taken at a variety of oblique angle couch and gantry positions.

200 The head phantom was then attached to the end treatment couch using the halo screws as done for the CT scan. In-room wall lasers were used to assist the initial raw visual set-up in positioning the center of the head phantom roughly to the isocenter. Set-up of the head phantom detailing the position of the gel vial inside is depicted in Figure 2. 


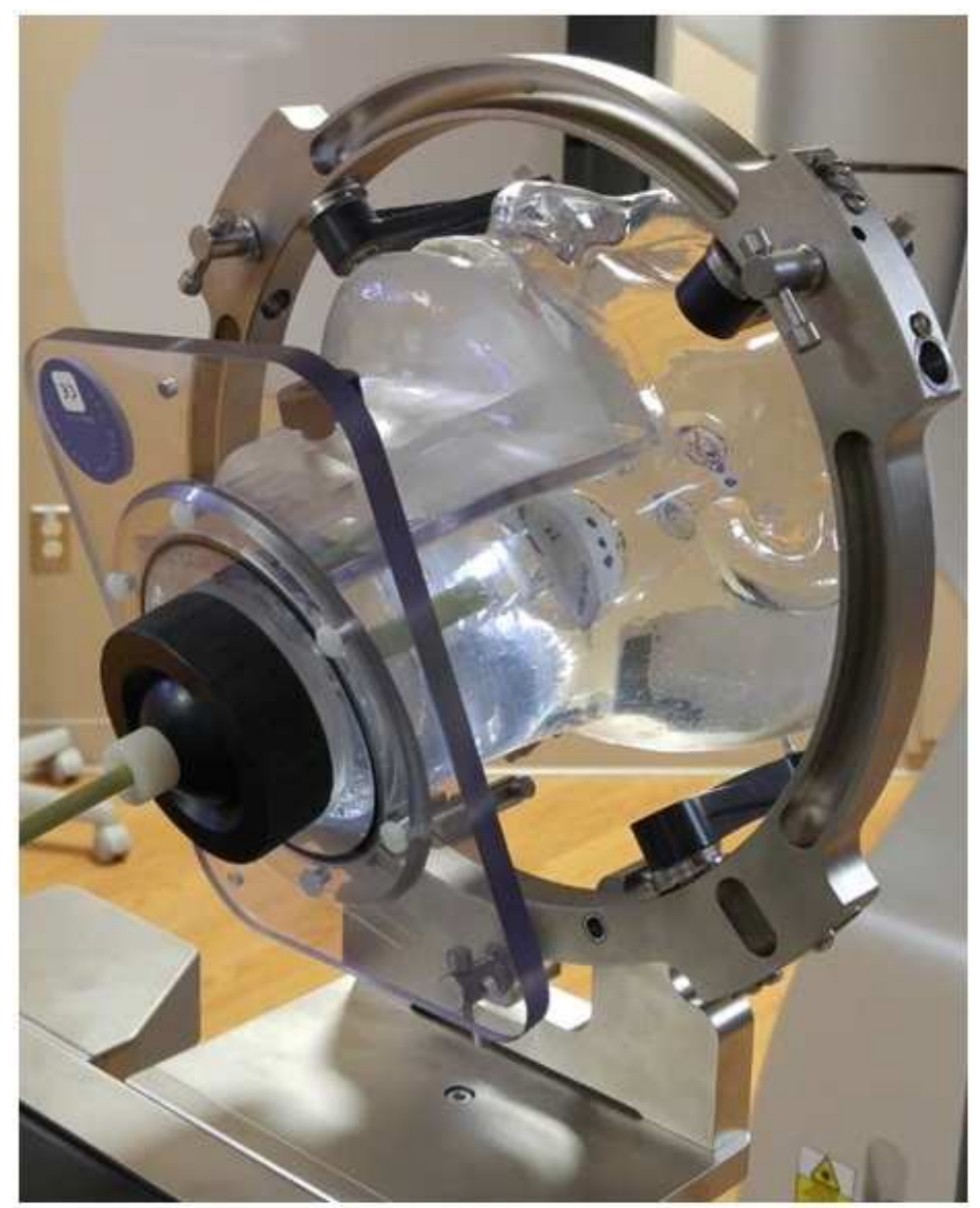

Figure 2. Stereotactic radiosurgery head frame mounted to RSVP Phantom II with ClearView ${ }^{\circledR}$ gel vial present as target. The rod is shown through the neck lock, connected to the adaptor, which is glued to the gel vial lid

A CBCT scan at $120 \mathrm{kVp}$ was then conducted. The CBCT image data set was registered to the CT data set used for planning. Shifts in the position of the couch were then conducted automatically at the console,

210 whereby the end result is a repositioning of the head phantom such that the surface of the head is precisely in the same 6DOF orientation as planned. This alignment coincides with the centroid of the gel vial being then set precisely at isocenter. At this point, the cone mounting base, the $15 \mathrm{~mm}$ cone, and the security clamp were connected to the collimator. One by one, each SRS arc beam was delivered using the corresponding couch rotation position and angular gantry arc start and stop positions. Upon

215 completion of radiation delivery, the head phantom was removed from the treatment vault. The locking 
mechanism at the neck of the head phantom was carefully unlocked, permitting the release of the rod, adaptor, and gel vial assembly. After the adaptor was unscrewed from the rod, the remaining gel vial and affixed adaptor were permitted to dry before being shipped back to the manufacturer for scanning. The gels were scanned two days after irradiation at the manufacturer's facility.

\section{RESULTS}

Appearance of the purple-colored formazan dye was evident immediately after radiation. A darker hue of dye concentrated at the center of the vial agreed with our expectations. ${ }^{29}$ Proof of color change in the

225 gel is evident in Figure 3, where the purple haze appears at the center of the vial. Dosimetry results were first compared between the plan and the measured scan for the three principal planes of view: axial, coronal and sagittal.

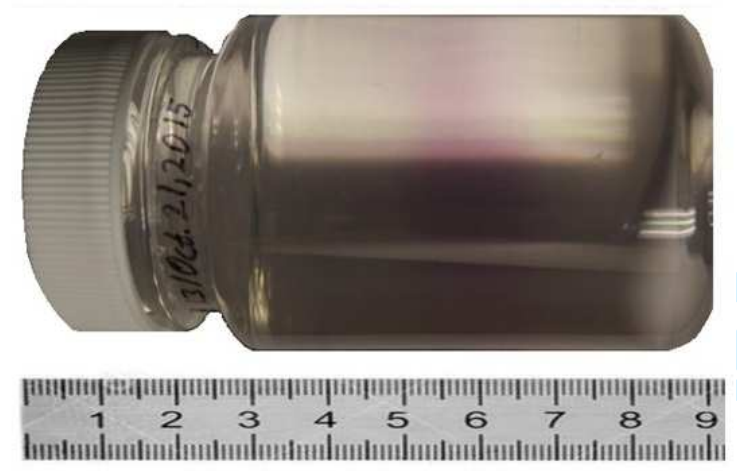

Figure 3. ClearView $^{\circledR}$ gel color change after irradiation

Shown in Figure 4 are planar dose cloud views for each. Planned results are shown to the left with corresponding optically-scanned results to the right. The distinct shape of the vial is noted from observed attenuation by the glass wall during scanning at the right. The red shaped cloud distinguishes the high dose level planned and delivered from the blue low dose level background. Plane comparisons 
235 reveal a marked similarity in the geometric location of highest dose within the vial. ${ }^{30}$ From this point, the determination of the accuracy in delivery is all that remained.

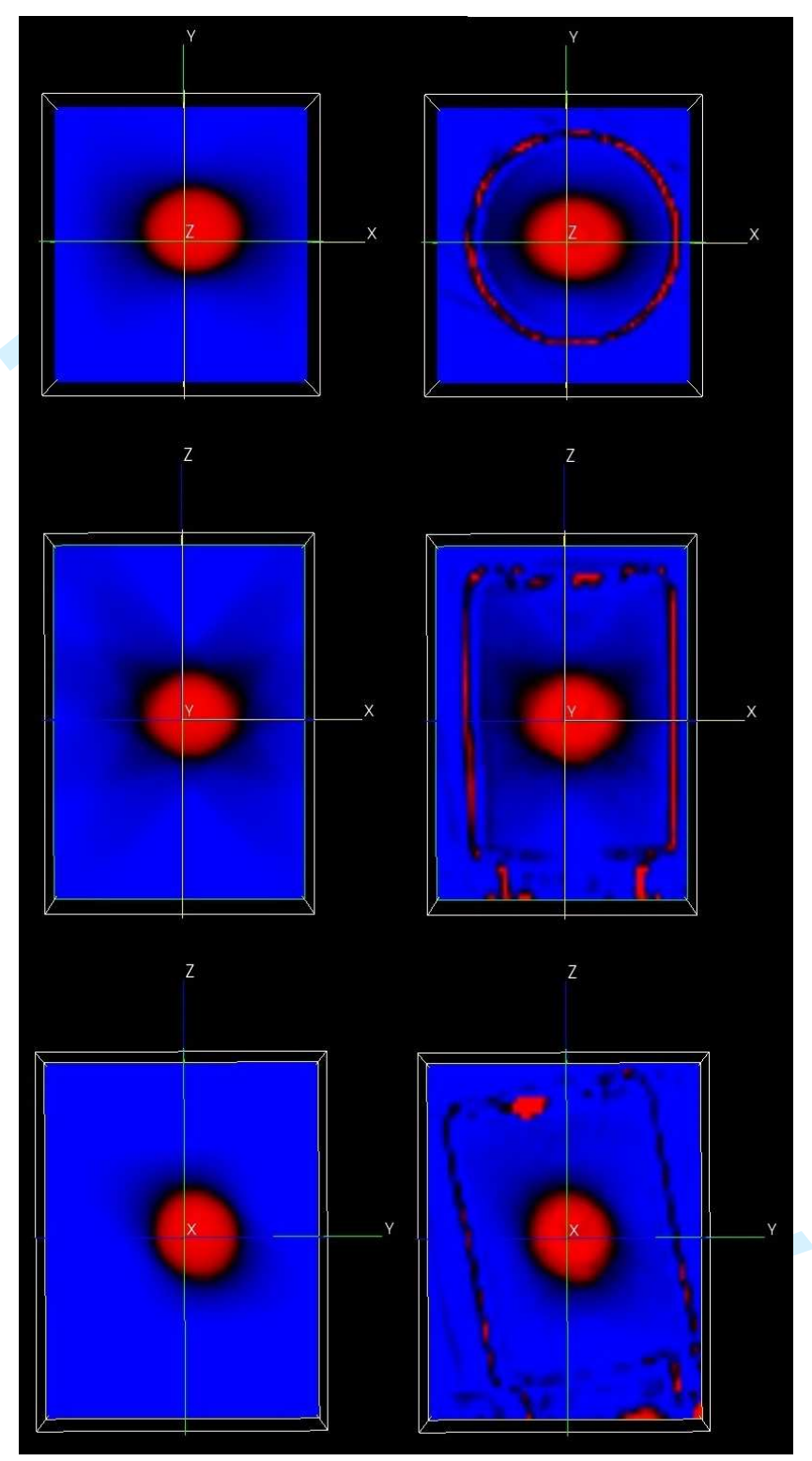

Figure 4. 2D planes detailing dose delivery isocenter geometry and distribution as planned (left) and measured (right); axial plane (XY), coronal plane (ZX), sagittal plane (ZY)

Tools within Vista ${ }^{\circledR}$ View software further permit comparisons in dose using cross-plots. Plots were generated from the wall of the vial through each axis for a direct comparison of dose planned and 
delivered across the positional length of the line. Figure 5 illustrates the line-dose profiles for each plane considered. The planned dose profile is depicted in blue, whereas the resulting optically measured dose 245 profiles from the gel are depicted in red. Error bars for the measured data reflect the uncertainty $(\delta)$ in delivery as determined by the square root of the sum of the squares of each uncertainty. Contributions to systematic errors were considered in similar to those published by the AAPM Task Group- $42 .{ }^{31}$ The net uncertainty as determined by quadrature was $\delta=1.6 \mathrm{~mm}$. In these positional plots, the error bars indicate the allowable range to which delivery is tolerated to vary upon measurement. Given that the 250 error bar width on measured data for each plane envelopes the expected planned plot, it is concluded that the dose delivery was within the window of acceptable accuracy. A dialog of uncertainty types follows for completeness. 

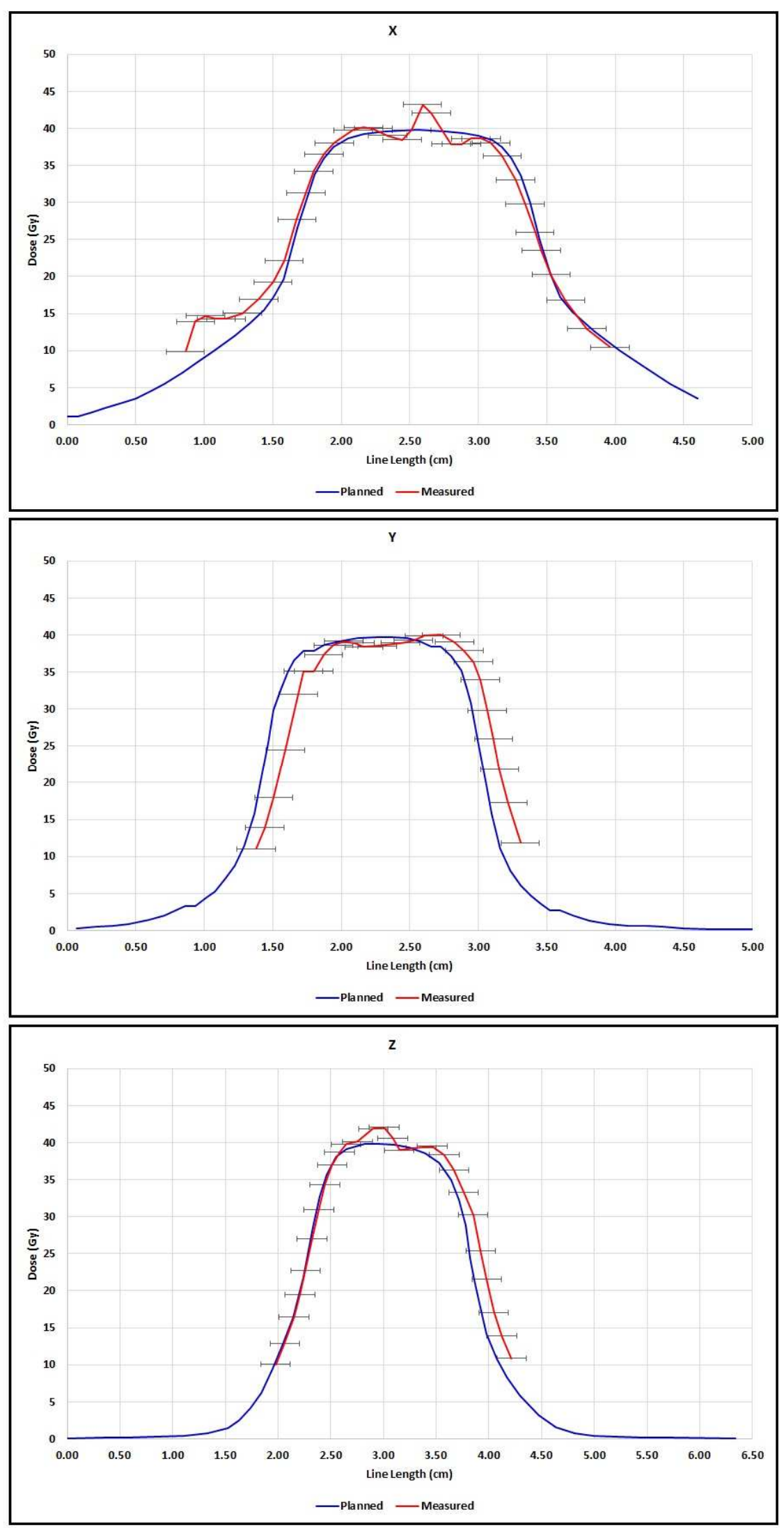
Figure 5. Line-dose profiles for all planes; axial plane (top), coronal plane (middle), sagittal plane (bottom)

\section{DISCUSSION}

We accounted for five systematic uncertainties in this research. First, there was an observed potential inaccuracy in the manual alignment of the acrylic adaptor. As stated earlier, we affixed a vial-rod adaptor to the plastic vial in order to make it possible to connect to the RSVP Phantom II rod. We knew

260 that each ClearView ${ }^{\circledR}$ gel vial was scanned prior to irradiation in order to establish a baseline. At that time, the vial-rod adaptor was attached to the gel vial. It was later disconnected during transit. Upon reattachment, the re-alignment was made possible by delineated $1.0 \mathrm{~mm}$ wide marker lines. Although we made every effort possible to reduce misalignment by matching the center of the lines marked, the uncertainty of $\delta_{1}=1.0 \mathrm{~mm}$ was noted as possible.

During linear accelerator QA, the Winston-Lutz test revealed that the combination of gantry and couch rotations provide for an additional $\delta_{2}=0.6 \mathrm{~mm}$ in isocenter displacement. Given that the treatment plan called for both gantry and couch rotational deliveries, this uncertainty must be included. Prior to dose delivery, we made use of a $\mathrm{CBCT}$ registration technique to match the external surface geometry of the

270 phantom in the treatment vault to that of the plan. The uncertainty in $\mathrm{CBCT}$ registration is the specification of the manufacturer at $\delta_{3}=0.1 \mathrm{~mm}$ for a 6DOF couch.

Alignment of the Vista ${ }^{\circledR}$ scanner is crucial for scanning. It should be identical during baseline measurements as it was used for delivery measurements. To ensure uniformity, small vial markings

275 require realignment. The width of the marks on the vial and scanner are smaller in comparison to those applied to the rod-vial adaptor. Nevertheless, the vial realignment to the scanner does indicate an uncertainty of $\delta_{4}=0.5 \mathrm{~mm}$. Finally, the more complex inaccuracy issue to address is that of the Vista ${ }^{\circledR}$ 
scanner readout and calibration. There is a refractive index mismatch between the jar contents and the jar wall, which becomes an issue for optical clarity. Different methods of molding and different mold designs lead to different artifacts. Consequently, the thicker the wall of the vial, the worse the aberrations appear. Additionally, for a thicker wall, we lose more information just inside the wall of the vial into the gel dosimeter itself. Therefore, we chose to accept the best-case scenario for testing, which included a vial with wall thickness of $1.0 \mathrm{~mm}$. Still, with hundreds of histories available for this method, we uncovered an underlying uncertainty in positional calibration up to $1.0 \mathrm{~mm}$ over time. The readout of 285 the $\operatorname{Vista}^{\circledR}$ scanner then contributes a possible uncertainty of $\delta_{5}=1.0 \mathrm{~mm}$. The overall uncertainty is then calculated as $\delta_{\mathrm{x}}=1.6 \mathrm{~mm}$ by quadrature. After consideration of the uncertainty within the delivery from endto-end, this information should be shared with the radiation oncologist for consideration in their definition of the clinical target volume (CTV) and margins assigned thereafter for the planning target volume (PTV), which define the treatment volume. A summary of these findings are presented in Table 1.

Table 1. Uncertainties in delivery and analysis

\begin{tabular}{lc}
\hline \hline Uncertainty type: & $\delta_{\mathrm{x}}(\mathrm{mm})$ \\
\hline Acrylic adapter manual alignment & 1.0 \\
Winston-Lutz isocenter test & 0.6 \\
CBCT registration & 0.1 \\
Vista $^{\circledR}$ View scanner alignment & 0.5 \\
Vista $^{\circledR}$ View scanner readout & 1.0 \\
\hline Standard deviation by quadrature $(\delta)$ & 1.6 \\
\hline \hline
\end{tabular}

The RSVP Phantom II proved to be a cost-effective and easy to use phantom for such testing. We were able to conclude that the study could easily accommodate a mask-based delivery similarly. For tight

295 fitting masks that are not removed from the head phantom during planning, we assume there to be no additional uncertainties necessary for incorporation. Even if bite-block testing is considered, CBCT automatic alignment will mitigate re-fitting issues with the phantom and bite tray. ${ }^{14}$ 


\section{CONCLUSIONS}

300 In our testing, we made use of the RSVP Phantom II head phantom and a halo mount, typical of SRS treatments. Fabrication of an adaptor made it possible to affix a vial containing ClearView $^{\circledR}$ gel dosimter inside the phantom to resemble a small target lesion. Employing an SRS treatment delivery, the ClearView $^{\circledR}$ gel discolored to a purple hue near the center of the vial. Vista ${ }^{\circledR}$ View software enabled a direct comparison of planned to optically scanned dosimetry. The geometric and dosimetric accuracy in

305 delivery was verified as all line-dose profile plots align within the window of positional error uncertainty.

In conclusion from this evaluation, we demonstrated that ClearView ${ }^{\circledR}$ gel contained in a vial is a suitable dosimeter for verifying geometric accuracy in delivery in radiation therapy. We recommend sites take advantage of the opportunity to employ more rigorous end-to-end testing as we have shown here. This

310 method is beneficial for stereotactic radiosurgery and radiotherapy delivery end-to-end quality assurance, but should also be found useful similarly for small-field volumetric modulated arc therapy (VMAT), intensity modulated radiation therapy (IMRT), and three-dimensional conformal radiation therapy (3DCRT).

\section{ACKNOWLEDGEMENTS}

The opportunity to study ClearView ${ }^{\circledR}$ gel was facilitated in cooperation with John Miller and Kalin I. Penev of Modus Medical Devices, Inc. ${ }^{\circledR}$ (Ontario, CANADA) who donated gels and scanner time to assist in data collection only. 
The authors have no relevant conflicts of interest to disclose. Gels and scanner time were donated free of charge by Modus Medical Devices, Inc. ${ }^{\circledR}$ (Ontario, CANADA).

\section{REFERENCES}

1. J.P. Bissonnette, P.A. Balter, L. Dong, K.M. Langen, D.M. Lovelock, M. Moften, D.J. Moseley, J.J. Sonke, S. Yoo. “Quality assurance for image-guided radiation therapy utilizing CT-based technologies: A report of the AAPM Task Group-179." Med. Phys. 39(4): 1946-1963 (2012).

2. L.J. Schreiner. "Review of Fricke gel dosimeters." J. Phys.: Conf. Ser. 3: 9-21 (2004).

3. C. Baldock, "Historical overview of the development of gel dosimetry: a personal perspective," Institute of Physics Publishing. J. Phys.: Conf. Ser. 56: 14-22 (2006).

4. L.E. Olsson, S. Petersson, L. Ahlgren, S. Mattsson. “Ferrous sulphate gels for determination of absorbed dose distributions using MRI technique: basic studies." Phys. Med. Biol. 34(1): 43-52 (1989).

5. C. Baldock, Y. De Deene, S. Doran, G. Ibbott, A. Jirasek, M. Lepage, K.B. McAuley, M. Oldham, L.J. Schreiner. "Topical Review: Polymer gel dosimetry." Phys. Med. Biol. 55(5): R1-R63 (2010).

6. J. Novotny, P. Dvorak, V. Spevacek, J. Tintera, J. Novotny, T. Cechak, R. Liscak. “Quality control of the stereotactic radiosurgery procedure with polymer gel dosimetry." Radiother. Oncol. 63: 223230 (2002).

7. V. Cosgrove, P.S. Murphy, M. McJury, E.J. Adams, A.P. Warrington, M.O. Leach, S. Webb. "The reproducibility of polyacrylamide gel dosimetry applied to stereotactic conformal radiotherapy." Phys. Med. Biol. 45: 1195-1210 (2000). 
345 8. K. McAuley. "The chemistry and physics of polyacrylamide gel dosimeters: why they do and don't work." J. Phys.: Conf. Ser. 3: 1 (2004).

9. Y. De Deene, K. Vergote, C. Claeys, C. De Wagter. "The fundamental radiation properties of normoxic polymer gel dosimeters: a comparison between a methacrylic acid based gel and acrylamide based gels." Phys. Med. Biol. 51(3): 653-673 (2006).

350 10. R. Senden, P. De Jean, K.B. McAuley, L.J. Schreiner. "Polymer gel dosimeters with reduced toxicity: a preliminary investigation of the NMR and optical dose-response using different monomers." Phys. Med. Biol. 51(14): 3301-3314 (2006).

11. H. Fricke, S. Morse, "The chemical action of Roentgen rays on dilute ferrous sulphate solutions as a measure of radiation dose," Am. J. Roentgenol. Radium Therpay. Nucl. Med. 18: 430-432 355 (1927).

12. H. Fricke, E.J. Hart, Chemical Dosimetry, in F.H. Attix and W.C. Roesch (Eds.), "Radiation Dosimetry" (2 ${ }^{\text {nd }}$ ed.), Vol. 2, Chap. 12 (Academic Press, New York, 1966).

13. L.J. Schreiner, T. Olding, "Gel dosimetry," Seminar: AAPM Summer School - Clinical dosimetry measurements in radiotherapy (2009).

360 14. R.M. Fried. "Optical imaging of radiation dose distributions in a ferrous-gelatin-xylenol orange gel dosimeter." Rutgers, The State University of New Jersey, (UMI, Ann Arbor, 1995).

15. A. Beer. "Determination of the absorption of red light in colored liquids." Annalen. der Physik. und Chemie. 86: 78-88 (1852).

16. A.K. Pikaev, Z.K. Kriminskaya. "A new dosimetric system based on aqueous alcoholic solutions of tetrazolium salt," Mendeleev Comm. 5(5): 200-201 (1995).

17. A.K. Pikaev, Z.K. Kriminskaya. "Use of tetrazolium salts in dosimetry of ionizing radiation." Rad. Phys. and Chem. 52(1): 555-561 (1998). 
18. T. Olding, J. Darko, L.J. Schriner. "Effective management of FXG gel dosimetry," J. Phys.: Conf. Ser. 250: 012028 (2010).

370 19. T. Olding, O. Holmes, L.J. Schriner. "Cone beam optical computed tomography for gel dosimetry I: scanner characterization," Phys. Med. Biol. 55: 2819-2840 (2010).

20. E. Pappas, T.G. Maris, S. Manolopoulos, F. Zacharopoulou, A. Papadakis, S. Green, C. Wojnecki, "Stereotactic radiosurgery photon field profile dosimetry using conventional dosimeters and polymer gel dosimetry. Analysis and inter-comparison." $5^{\text {th }}$ International Conference on Radiotherapy Gel Dosimetry (DOSGEL 2008) 164: 1-4 (2009).

21. C. Coffey, M. Sanders, K. Cashon, R. Miller, J. Walsh, P. Patel. "A tissue equivalent phantom for stereotactic radiosurgery localization and dose verification," Stereotact. Functional. Neurosurg. 61(S1): 130-141 (1993).

22. M.S. Gossman, J.R. Levy JR. "Strategic radiosurgery head phantom bite tray fit reproducibility." J. 380 Med. Dev. 8: 3 (2014).

23. R.S. Hong, J.M. Kartush. "Acoustic neuroma neurophysiologic correlates: facial and recurrent laryngeal nerves before, during, and after surgery." Otolaryngol. Clin. North Am. 45(2): 291-306 (2012).

24. R. Ramaseshan, M. Heydarian. "Comprehensive quality assurance for stereotactic radiosurgery treatments." Phys. Med. Biol. 48(14): N199-N205 (2003).

25. W. Mao, L. Lee, L. Xing. "Development of a QA phantom and automated analysis tool for geometric quality assurance of on-board MV and kV x-ray imaging systems." Med. Phys. 35(4): 1497-506 (2008).

26. M.B. Sharpe, D.J. Moseley, T.G. Purdie, M. Islam, J.H. Siewerdsen, D.A. Jaffray. "The stability of mechanical calibration for a $\mathrm{kV}$ cone beam computed tomography system integrated with linear accelerator." Med. Phys. 33(1): 136-44 (2006). 
27. W. Lutz, K.R. Winston, N. Malek. "A system for stereotactic radiosurgery with a linear accelerator." Int. J. Radiat. Oncol. Biol. Phys. 14(2): 373-381 (1988).

28. International Electrotechnical Commission (IEC). "Radiotherapy equipment - coordinates, 395 movements and scales." IEC 61217: 1-146 (2008).

29. A. Appleby and A. Leghrouz. "Imaging of radiation dose by visible color development in ferrous agarous xylenol-orange gels." Med. Phys. 18: 309-312 (1991).

30. J. Hazle, L. Hefner, C. Nyerick, L. Wilson, A.L. Boyer. “Dose response characteristics of a ferroussulphate-doped gelatin system for determining radiation absorbed dose distributions by magnetic resonance imaging (FeMRI)." Phys. Med. Biol. 36: 1117-1125 (1991).

31. M.C. Schell, F.J. Bova, D.A. Larson, D.D. Leavitt, W.R. Lutz, E.B. Bodgorsak, A. Wu. “Stereotactic radiosurgery: AAPM Report 54 of Task Group 42." (American Association of Physicists in Medicine, College Park, MD, 1995). 